3 Caw ley R, Mauldin R D. Multifractal decompositions of Moran Fractals. Adv in M ath, 1992, 92: 196 236

4 Bandt C. Deterministic fractals and fractal measures. Rendt Istit M at Vniv Trieste, 1993, 23(1): 1 40

5 Cajar H. Billingsley dimension in probability space. Lect Notes in Math, 1981, Vol 892

\title{
Au_Pt_Ni/p_InP 新的低欧姆接触电极
}

\author{
李秉臣 彭 晔 李建中 \\ (中国科学院半导体研究所国家光电子工艺中心, 北京 100083)
}

摘要 用磁控溅射法淀积的非合金膜系 $\mathrm{Au} \_\mathrm{Pt} \_\mathrm{Ni} / \mathrm{p} \_\mathrm{InP}\left(1 \sim 2 \times 10^{18} \mathrm{~cm}^{-3}\right)$, 在 $400{ }^{\circ} \mathrm{C} 30 \mathrm{~s}$ 的 退火条件下, 实现了比接触电阻低达 $3 \times 10^{-6} \Omega \cdot \mathrm{cm}^{2}$ 的欧姆接触. 对不同温度 $\left(300 \sim 500{ }^{\circ} \mathrm{C}\right)$ 退火的 Au_Pt_Ni/p_InP 欧姆接触所做的 AES 深度剖面分析揭示了 Pt 膜层阻挡 Au 的内扩散 和 $\mathrm{InP}$ 外扩散的作用.

\section{关键词 磁控溅射 快速热退火 比接触电阻 AES 深度剖面分析}

在 InP 化合物半导体器件制备过程中, $\mathrm{p}$ 型欧姆接触要比 $\mathrm{n}$ 型难做得多. 其原因一部分 是由材料本身特性决定的, 空穴的有效质量要比电子的有效质量大得多, 势垒高度也比 $\mathrm{n}$ 型材 料高 $\left(\mathrm{n} \_\mathrm{InP}\right.$ 的势垒高度 $\Phi_{\mathrm{Bn}}$ 为 $0.4 \mathrm{eV}$, 而 $\mathrm{p} \_\mathrm{InP}$ 的 $\Phi_{\mathrm{Bp}}$ 为 $\left.0.97 \mathrm{eV}\right)$. 另一部分原因是制备欧 姆接触工艺本身的问题. 现在比较常见的 $\mathrm{Au} Z \mathrm{Zn}, \mathrm{Au}$ B Be 合金膜系 ${ }^{[1.3 .41}, \mathrm{Zn}$ 不仅与 GaAs, $\mathrm{InP}$ 粘附性差, 而且由于 $\mathrm{Zn}$ 具有较高的蒸气压, 在 $\mathrm{Au}$. Zn 蒸发过程中很难控制 $\mathrm{Zn}$ 的比例. Be 的蒸气压与 $\mathrm{Au}$ 差不多, 是很好的掺杂剂, 但它的缺点是具有毒性, 对人体有害. 另外 $\mathrm{Au}$ 是快 扩散杂质, 合金退火时, Au 很容易扩散到 P_N 结内, 使结特性变坏.

$\mathrm{Ti}$ _Pt_Au 非合金膜系能较好地解决上述问题 ${ }^{[5]}$, 但是它必须要求 $\mathrm{p}$ 型材料表面浓度大于 $10^{19} \mathrm{~cm}^{-3}$ 时, 才具有低的比接触电阻 $\left(6 \times 10^{-6} \Omega \cdot \mathrm{cm}^{2}\right)$. 这一要求, 对 p_InP 来说, 掺杂后的表 面浓度很难达到 $10^{19} \mathrm{~cm}^{-3}$. 因此, 在一般情况下, Au_Pt_Ti/p_InP(表面浓度为 $5 \times 10^{18} \mathrm{~cm}^{-3}$ 时) 在比接触电阻只能是 $10^{-4} \Omega \cdot \mathrm{cm}^{2}$ 数量级.

本文所论述的是采用 $\mathrm{Au}$ _Pt_Ni/p_InP 非合金膜系. 在 $\mathrm{p} \_\mathrm{InP}$ ( 掺 $\mathrm{Zn}$, 掺杂浓度为 $1 \sim 2 \times$ $10^{18} \mathrm{~cm}^{-3}$ ) 基片上, 进行了比接触电阻的试验研究, 并在实际的激光器制备中进行了 $\mathrm{Au} \mathrm{Pt}_{-}$ $\mathrm{Ni} / \mathrm{p}^{+}$InGaAsP/n_InP 的应用研究.

1 实验

实验研究所用的设备是美国 INNOTEC 公司生产的 VS_24C 多靶磁控溅射系统. Ni 靶、 $\mathrm{Au}$ 靶的纯度为 $99.999 \%, \mathrm{Pt}$ 靶纯度为 $99.99 \%$ 。

首先对 p_InP( 掺 $\mathrm{Zn}, 1 \sim 2 \times 18^{18} \mathrm{~cm}^{-3}$ ) 基片进行清洗, 然后制备 TLM 法(传输线法) 测试 图形. 接着用 $\mathrm{HCl}: \mathrm{H}_{2} \mathrm{O}=1: 5$ 溶液漂洗 $30 \mathrm{~s}$, 去除 $\mathrm{Na}^{+}, \mathrm{K}^{+}$等金属离子的污染, 并用去离子 水冲洗, 干 $\mathrm{N}_{2}$ 吹干.

为了做比较实验, 在镀膜前分别用两种方法去除自然氧化层, 一种方法用 $\mathrm{Ar}^{+}$轰击清洗 ${ }^{[2]}$ 
$(100 \mathrm{~W}, 30 \mathrm{~s})$, 另一种方法用 $\mathrm{CH}_{4}+\mathrm{H}_{2}$ 干法清洗 $(100 \mathrm{~W}, 30 \mathrm{~s})$, 然后立即放入真空室内抽真 空. 使本底真空度降低到 $1.33 \sim 2.66 \times 10^{-5} \mathrm{~Pa}$, 冲入 $99.999 \%$ 高纯 $\mathrm{Ar}$ 气, 在工作压力为 $2.66 \mathrm{~Pa}$ 的条件下, 分别溅射 $\mathrm{Ni}$ 膜 $(50 \mathrm{~nm}) 、 \mathrm{Pt}$ 膜 $(100 \mathrm{~nm}) 、 \mathrm{Au}$ 膜 $(100 \mathrm{~nm})$. 取出样品并剥离 出 TLM 法所需的图形. 然后用 706 厂生产的白光快速热退火设备进行快速退火试验, 其温 度分别为 $300,350,400,450,500{ }^{\circ} \mathrm{C}$, 退火时间为 $30 \mathrm{~s}$. 接着用三探针传输线法测量其比接 触电阻. 找出最佳的退火条件, 最后用 AES 深度剖面分析法观察不同退火条件下, Au_Pt_Ni 各膜层组分和 In, P 组分在 P_InP 界面的分布变化.

\section{2 结果与讨论}

\section{1 比接触电阻 $\rho_{c}$}

对欧姆接触质量的评价, 一般用比接触电阻 $\rho_{\mathrm{c}}$ 来表征. 三探针传输线法是测量 $\rho_{\mathrm{c}}$ 的常 用方法. 我们使用的是 TLM 一型全自动电阻测量仪, 其测量结果如图 1 所示.

从图 1 中, 可以看到, 在退火温度为 $300{ }^{\circ} \mathrm{C}$, 时间为 $30 \mathrm{~s}$ 时, $\rho_{\mathrm{c}}$ 为 $10^{-4}$ 或 $3 \times 10^{-3} \Omega \cdot \mathrm{cm}^{2}$. 但 在退火温度为 $400{ }^{\circ} \mathrm{C}$, 时间为 $30 \mathrm{~s}$ 时, 比接触电阻 降低到 $3 \times 10^{-6} \Omega \bullet \mathrm{cm}^{2}$. 此后随着退火温度的提 高, 比接触电阻反而增大, 在退火温度 $450{ }^{\circ} \mathrm{C}$, 时 间为 $30 \mathrm{~s}$ 时, $\rho_{\mathrm{c}}$ 仍在 $10^{-6}$ 数量级范围内 $\left(9 \times 10^{-6}\right.$ 或 $\left.6 \times 10^{-6} \Omega \cdot \mathrm{cm}^{2}\right)$. 但在退火温度为 $500{ }^{\circ} \mathrm{C}$, 时 间为 $30 \mathrm{~s}$ 时, $\rho_{\mathrm{c}}$ 升为 $1 \times 10^{-5} \Omega \bullet \mathrm{cm}^{2}$ 左右.

对这一实验结果, 归纳为以下 3 个值得注意 的方面:

(1) 对 p_InP 表面的严格清洗, 确保 InP 表面 无自然氧化层. 这可以用 AES 深度剖面分析法来

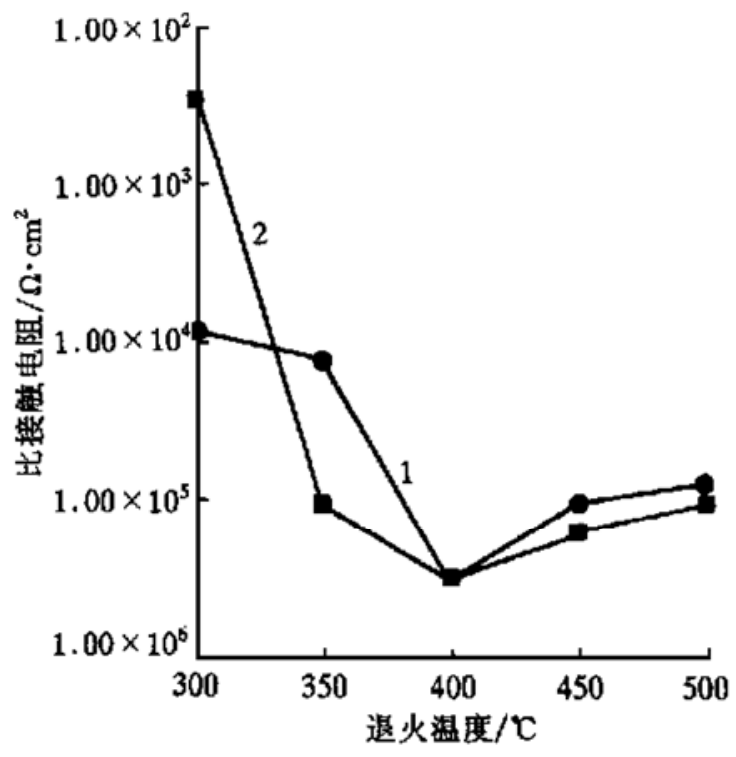
检测. 采用两种方法来保证 $\mathrm{InP}$ 表面的清洁度.

（ ）用 $\mathrm{Ar}^{+}$轰击 $(100 \mathrm{~W}, 30 \mathrm{~s})$ 清洗. ( ) 用 $\mathrm{CH}_{4}+$ $\mathrm{H}_{2}$ 干法清洗( $\left.100 \mathrm{~W}, 30 \mathrm{~s}\right)$. 实验结果由图 1 表 明. 用 $\mathrm{CH}_{4}+\mathrm{H}_{2}$ 清洗, 其效果更好一些.

(2) $\mathrm{Ni}$ 膜不含氧是很重要的一个环节. 为了
图 1 Au_Pt_Ni/P_InP 的比接触电阻与退火温度 的关系

1 一一用 $\mathrm{Ar}^{+}$轰击清洗 $(100 \mathrm{~W}, 30 \mathrm{~s}), 2$ 一用 $\mathrm{CH}_{4}+$ $\mathrm{H}_{2}$ 干法清洗( $100 \mathrm{~W}, 30 \mathrm{~s}$ ) 实现 $\mathrm{Ni}$ 膜层内不含氧, 采用的方法是一方面把本底真空度尽量地提高, 其系统真空度降低到 $1.33 \sim 2.66 \times 10^{-5} \mathrm{~Pa}$. 另一方面, 为使 $\mathrm{Ar}$ 气的纯度尽量提高, 本实验使用的是 $99.999 \%$ 纯度 的 Ar. 在这基础上还要进行 $\mathrm{Ni}$ 的予溅射. 这也可以用来提高真空室背景 $\mathrm{Ar}$ 的纯度, 因为溅 射出来的 $\mathrm{Ni}$ 分子或原子团可以与真空室内的残余气体如 $\mathrm{O}_{2}, \mathrm{H}_{2} \mathrm{O}$ 等反应形成 $\mathrm{Ni}_{x} \mathrm{O}_{y}$ 淀积在 挡板上. 用 $\mathrm{Si}$ 表面贼射淀积 $\mathrm{Ni}$ 来做实验, 根据 $\mathrm{Ni} / \mathrm{Si}$ 界面的 AES 深度剖面分析 $\mathrm{Ni}$ 膜组分是 否含氧来确定予溅射时间.

在予溅射功率 $240 \mathrm{~W}$, 时间为 $30 \mathrm{~min}$ 时, $\mathrm{Ni} / \mathrm{Si}$ 的 $\mathrm{AES}$ 深度剖面分析图表明在金属 $\mathrm{Ni}$ 层 中, 仍有少量 $\mathrm{O}_{2}$ 存在. 在相同的溅射功率条件下, 把予溅射时间增加到 $45 \mathrm{~min}$, 所进行的 $\mathrm{Ni} /$ $\mathrm{Si}$ 的 AES 深度剖面分析表明, 在金属 $\mathrm{Ni}$ 层中已几乎没有 $\mathrm{O}_{2}$, 其结果如图 2 所示. 
（3）快速热退火. 合金退火对于形成欧 姆接触来讲是相当重要的步骤, 在热退火过程 中, 升温和降温的速度以快为好. 加热快的优 点是在加热过程中, 如果温度即使是超过合金 熔点, 由于来不及熔化和聚球, 也容易获得好 的表面形貌, 使器件的可靠性得到了改善和提 高. 快速降温比快速升温更重要．如将合金 面倒置在 $\mathrm{Cu}$ 热沉上快速降温, 可使欧姆接触 改善 2 7 倍. 我们所选择的合金退火温度为 $300 \sim 500{ }^{\circ} \mathrm{C}$ 温度范围内, 每隔 $50{ }^{\circ} \mathrm{C}$ 做一次试 验, 退火时间为 $30 \mathrm{~s}$.

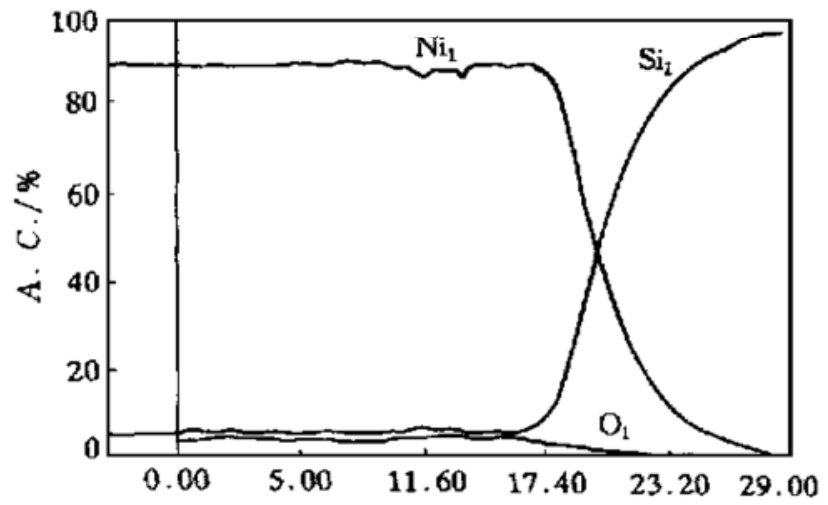

\section{2 不同退火温度下 Au_Pt_Ni/p_InP 的 AES} 深度剖面分析

退火温度为 $300{ }^{\circ} \mathrm{C}$ 时间为 $30 \mathrm{~s}$ 条件下的 AES 深度剖面分析图表明, 在 Au_Pt_Ni/p_InP 界 面处, $\mathrm{Ni}$ 已经扩散到 $\mathrm{InP}$ 表面内, $\mathrm{Pt}$ 有效地阻挡了 $\mathrm{Au}$ 向 $\mathrm{InP}$ 的内扩散和 $\mathrm{In}$ 的外扩散. 与 TLM 法测量的比接触电阻相对照. 此时的 $\rho_{\mathrm{c}}$ 为 $10^{-4} \Omega \cdot \mathrm{cm}^{2}$ 数量级.

从温度为 $350^{\circ} \mathrm{C}$ 时间为 $30 \mathrm{~s}$ 的退火条件下的 $\mathrm{AES}$ 深度剖面分析图中可以看到, $\mathrm{Ni}$ 在 $\mathrm{InP}$ 表层内的含量增加, $\mathrm{Pt}$ 层有效地阻挡了 $\mathrm{In}$ 的外扩散和 $\mathrm{Au}$ 的内扩散, 此时的比接触电阻 $\rho_{\mathrm{c}}$ 为 $10^{-5}$ 数量级.

在温度为 $400{ }^{\circ} \mathrm{C}$ 时间为 $30 \mathrm{~s}$ 的退火条件 下, Au_Pt_Ni/p_InP 界面处各组分的分布变化 情况如图 3 所示. $\mathrm{Ni}$ 进一步向 $\mathrm{InP}$ 体内推 移, $\mathrm{Ni}$ 在 $\mathrm{InP}$ 表面的含量也随之增加. Pt 层 仍有效地阻挡 $\mathrm{Au}$ 的内扩散和 In 的外扩散. 此时的比接触电阻为 $3 \times 10^{-6} \Omega \mathrm{cm}^{2}$.

在温度为 $450{ }^{\circ} \mathrm{C}$ 时间为 $30 \mathrm{~s}$ 的退火条件 下, AES 深度剖面分析图表明, Au 在 $\mathrm{Ni}$ 层和 $\mathrm{InP}$ 表面内含量略有增加, In 的外扩散也渐渐 地表现出来, 可能是由于易老化的 $\mathrm{Au}_{x} \mathrm{In}_{y}$ 低

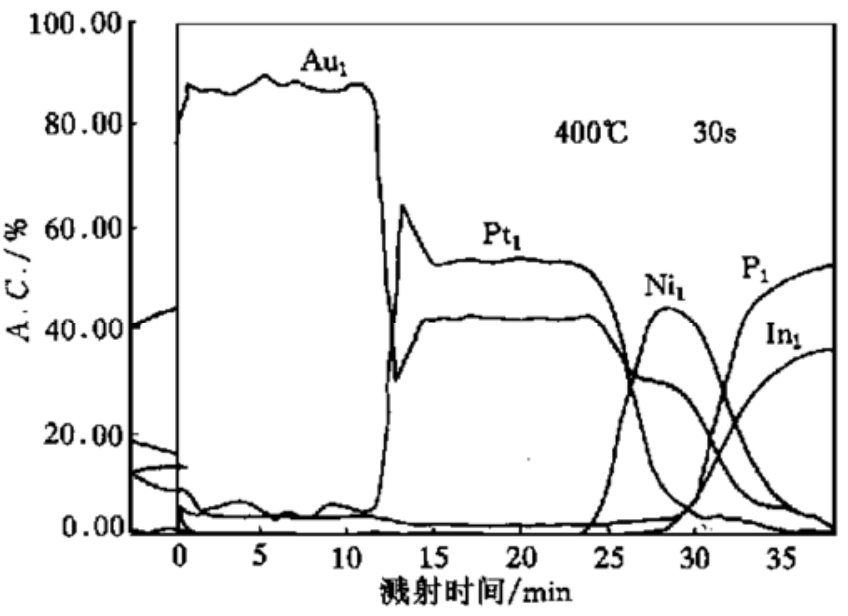
熔点金属化合物的少许形成 ${ }^{[6]}$, 比接触电阻 也略有增加, 其阻值为 $6 \sim 9 \times 10^{-6} \Omega \cdot \mathrm{cm}^{2}$.

从温度为 $500{ }^{\circ} \mathrm{C}$ 时间为 $30 \mathrm{~s}$ 的退火条件

图 $2240 \mathrm{~W}$ 予溅射 $40 \mathrm{~min}$ 后的 $\mathrm{Ni} / \mathrm{Si}$ AES 深度剖 面分析图 
$\mathrm{H}_{2}$ 处理的样片做了比较. 将两种样片一起溅射淀积 $\mathrm{Ni}(50 \mathrm{~nm}), \operatorname{Pt}(100 \mathrm{~nm}), \mathrm{Au}(100 \mathrm{~nm})$. 膜层在 $400{ }^{\circ} \mathrm{C}$ 温度下退火 $30 \mathrm{~s}$, 测量的串联电阻都为 $1 \Omega$ 左右.

另外, 在相同的 $\mathrm{p}^{+} \_\mathrm{InGaAsP} / \mathrm{n} \_\mathrm{InP}$ 基片与 $\mathrm{Au}$ _ Zn_Au p 型电极做比较, Au_Zn_Au 电极的串联 电阻 $R_{\mathrm{s}}$ 为 $1.5 \Omega$ 左右, NiPt_Au 电极的串联电阻为 $0.75 \Omega$ 左右, 串联电阻几乎小近一倍.

\section{3 结论}

根据以上实验结果, 可以得出以下结论:

(1) 采用磁控溅射法淀积的 $\mathrm{Au}$ _Pt_Ni/p_InP( 掺 $\mathrm{Zn}, 1 \sim 2 \times 10^{18} \mathrm{~cm}^{-3}$ ) 在退火温度 $400{ }^{\circ} \mathrm{C}$, 时间 $30 \mathrm{~s}$ 的条件下, 实现了低阻欧姆接触, 其比接触电阻为 $3 \times 10^{-6} \Omega \bullet \mathrm{cm}^{2}$.

(2) 不同的退火温度 $\left(300 \sim 500{ }^{\circ} \mathrm{C}\right)$ 的 $\mathrm{AES}$ 深度剖面分析表明, $\mathrm{Pt}$ 层阻挡 $\mathrm{Au}$ 的内扩散和 In 外扩散的作用, 但比接触电阻降低到 $3 \times 10^{-6} \Omega \cdot \mathrm{cm}^{2}$ 的机理尚不十分清楚.

利用 Au_Pt_Ni/ $\mathrm{p}^{+} \_$InGaAsP/n_InP 在长波长 $(1.3 \sim 1.5 \mu \mathrm{m})$ 激光器制备上进行了应用研 究, 取得了满意的结果.

致谢在实验研究过程中, 得到了余觉觉研究员、崔玉德高级工程师、王维明高级工程师、范东华高级工程师 的大力帮助, 在此深表感谢.

\section{参 考 文 献}

1 Wang L C, Park M H, Deng F, et al. Ge/ Pd( Zn) ohmic contact scheme on p_InP based on the solid phase regrow th principle. Appl Phys Lett, 1995, 66(24): 3 310 3312

2 Ren F. Low resistance ohmic contacts on nitrogen ion bambared InP. Appl Phys Lett, 1994, 65( 17): 2 165 2 167

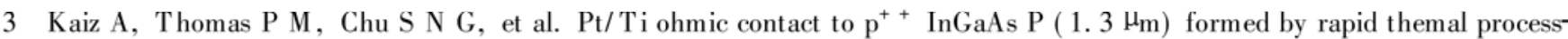
ing. J Appl Phys, 1990, 67(2) : 884 889

4 Lin X W, Liliental_Weber Z, Washbun J. Structural characterization of encapsulated Au/ Zn/ Au ohmic contacts to p_type GaAs. J V ac Sci Technol, 1993, B 11(1): 44 50

5 Hidenori Shimawaki, Naoki Furuhata, Kazuhiko Honjo. Ohmic contacts to p_GaAs with p ${ }^{+} / \mathrm{p}$ regrow n structures formed by metalorganic molecular beam epitaxy. J Appl Phys, 1991, 69(11): 7 939 7941

6 Barnard W O, Malherbe J B, Auret F D, et al. An Auger electron spectroscopy study of annealed gold contacts to InP. Thin Solid Films, 1992, 215: 42 49

\section{低于 $20 \mathrm{~nm}$ 的 $\mathbf{S}$ 元 $\mathbf{S}$ 光谱研究}

\section{杨治虎 马新文 王友德 刘惠萍 苏 宏 于得洋}

( 中国科学院近代物理研究所, 兰州 730000)

摘要 在兰州重离子加速器上采用束_箔光谱技术测量了 S Q 元 S 激发光谱, 经分析研究, 辨认出 30 多条光谱线. 这些谱线与理论及现有的一些实验结果相符合.

关键词 高电离态离子光谱 束箔方法

加速器上的硫元素光谱学的实验研究工作多数在低于 S 园电离级, 高于 $\mathrm{S}$ 园电离级的光谱 